\title{
Identifikation von Kovariableneinflüssen in der Füllungstherapie
}

\author{
Karl Schableger ${ }^{1}$, Notburga Pfoser ${ }^{1}$, und Helga Wagner ${ }^{2}$ \\ ${ }^{1}$ Abteilung Behandlungsökonomie, OÖ Gebietskrankenkasse Linz \\ ${ }^{2}$ Institut für Angewandte Statistik, Johannes-Kepler-Universität Linz
}

\begin{abstract}
Zusammenfassung: Um Informationen über die Haltbarkeit von Zahnfüllungen $\mathrm{zu}$ erhalten und den Einfluss verschiedener Faktoren zu untersuchen, wurden verschiedene statistische Analysen durchgeführt. Dafür standen in Oberösterreich Daten von rund 3.4 Millionen Zahnfüllungen aus den Jahren 1994 bis 2002 zur Verfügung. Neben einer Schätzung der Überlebensfunktion bzw. der Ausfallrate, wurde der Einfluss verschiedener Kovariablen auf die Lebensdauer der Füllungen in einem Cox-Modell geschätzt. Die im Verhältnis zur vorliegenden Datenmenge geringe Zahl von Kovariablen, legt es nahe, dass die Daten auch unbeobachtete Heterogenität enthalten. Von Interesse ist dabei insbesondere, ob diese auf Effekte der Leistungserbringer, d.h. der behandelnden Ärzte zurückgeführt werden können. Diese Effekte werden durch ein Gamma-Shared-Frailty-Modell geschätzt. Die Ergebnisse führen zu Vorschlägen einer differenzierteren Honorierung der Füllungstherapie.
\end{abstract}

\begin{abstract}
A survival analysis was carried out to get information about the durability of dental fillings and to investigate the influence of different factors. In Upper Austria data on about 3.4 million dental fillings made between 1994 and 2002 were available. In addition to estimating the survival and hazard function the effect of different covariables was analysed using a Cox-model. Compared to the large data set only a few covariates are observed, suggesting that unobserved heterogeneity might be present in the data. We are in particular interested whether part of this heterogeneity can be attributed to effects of the dentists. Their effects are estimated using a gamma frailty model. The results lead to proposals of differentiating payment for dental fillings.
\end{abstract}

Schlüsselwörter: Lebensdaueranalyse, Cox-Modell, Shared-Frailty-Modell, Zahnfüllungen.

\section{Einleitung}

Evidenzbasierte Medizin, Qualitätssicherung und Versorgungsforschung sind bedeutsame Ansätze in der Gesundheitsversorgung, die zunehmend an Akzeptanz gewinnen. Man darf davon ausgehen, dass sowohl die Erbringer medizinischer Dienstleistungen einerseits als auch die Krankenkassen als Financiers andererseits Interesse an einer Lebensdaueranalyse der von ihnen erbrachten bzw. zu finanzierenden Leistungen haben. Es gibt eine Vielzahl von Studien (vgl. die Übersicht klinischer Studien von Restaurationen im kaubelasteten Seitzahnbereich unter: www .zahnmedizin . spitta.biz/Fachinformationen/ Zahnmed._Fachgebiete/Füllungstherapie/ zur Haltbarkeit von Zahnfüllungen). 
Die Durchsicht der einschlägigen Literatur führt jedoch zu dem Ergebnis, dass die vorliegenden Untersuchungen

- keine ausreichende Berücksichtigung von Kovariablen aufweisen,

- unter praxisfremden Rahmenbedingungen (in Universitätskliniken o.ä.) ablaufen,

- wenige Patienten bzw. Füllungen umfassen,

- der Vergleich verschiedener Füllungsmaterialien im Vordergrund steht

- und nicht zuletzt meist nur Verfahren der deskriptiven Statistik bemühen und keine Lebensdaueranalysen erfolgen.

Die Füllungstherapie nimmt - betrachtet man Umsatz und Zahl der gelegten Füllungen - innerhalb des Leistungsspektrums der Zahnheilkunde eine absolute Spitzenstellung ein. So entfallen im Jahr 2004 27\% der Einzelleistungen bzw. knapp 50\% der Kosten des konservierend-chirurgischen Bereichs in der Zahnheilkunde im Rahmen der gesetzlichen Krankenversicherung in Österreich auf die Füllungstherapie (siehe Statistisches Handbuch der österreichischen Sozialversicherung, 2005). Es ist also naheliegend, die Frage nach der erwarteten Lebensdauer von Restaurationen, die unter täglichen Praxisbedingungen gelegt werden, zu stellen.

Unser besonderes Interesse galt der Analyse der Daten hinsichtlich

- Unterschieden in der Verweildauer von Füllungen unter soziodemografischen Merkmalen der Patienten (Geschlecht, Alter, Region)

- anderer Einflussgrößen wie Stamm-/Wechselpatient

- Unterschieden in der Verweildauer der Füllungen zwischen den Ärzten (Geschlecht, Alter, Region)

- sowie die Modellierung unbeobachteter Heterogenität als Arzteffekt unter Berücksichtigung von Kovariableneffekten.

\section{Daten}

Die Daten aus den neun Jahren 1994 bis 2002 stammen von der oberösterreichischen Gebietskrankenkasse. Mit rund 19 Millionen abgerechneten Leistungspositionen stellt dieser Datenbestand eine Herausforderung für die verwendeten Programme dar. Unter konservierend-chirurgische Zahnbehandlung fallen unter anderem Füllungen, Wurzelbehandlungen, Zahnentfernung und Röntgen, jedoch keine Kronen, Prothesen oder Zahnregulierungen. Nach erfolgter Plausibilitätskontrolle und Fehlerbereinigung wurde der Datensatz auf Patienten zwischen 15 und 65 Jahren eingeschränkt.

Jeder Zahn hat eine eindeutige zweistellige Nummer. Ober- und Unterkiefer sind in vier Quadranten unterteilt - die Zehnerstelle der Nummer eines Zahnes bezeichnet den Quadranten, in dem sich der Zahn befindet. Die Einerziffer ist die Nummer des Zahnes jeweils von der Mitte vom Schneidezahn weg nach hinten zum Weisheitszahn (siehe Abbildung 1). Um genau festlegen zu können, wo eine Füllung liegt und welche Bereiche des Zahnes sie überdeckt wird ein Zahn in 5 Flächen (Lokation M, O, D, B und L) unterteilt.

Für unsere Analyse haben wir den Datenbestand zusätzlich auf die Zähne 4 bis 8 eingeschränkt. Eine Schätzung der Verweildauer von Zahnfüllungen für die Zähne 1 bis 


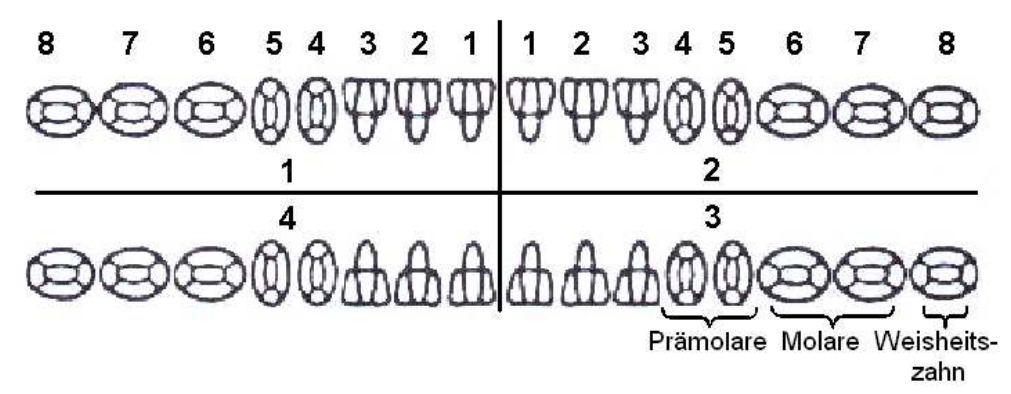

Abbildung 1: Zahnschema (Zahnnummerierung): 4 Quadranten, je 8 Zähne

3 ergibt wenig Sinn, handelt es sich doch um Frontzähne wo nicht nur unterschiedliche Füllungsmaterialien zum Einsatz kommen, sondern Füllungen auch häufig aus optischen Gründen ausgetauscht werden. Für Füllungen auf den kaubelasteten Molaren oder Prämolaren trifft dies hingegen kaum zu.

Neben einer halben Million Wurzelbehandlungen und Zahnentfernungen für die Bestimmung der Ausfallzeitpunkte blieben letztlich ca. 3.4 Millionen Füllungspositionen, für die eine Lebensdauer berechnet werden konnte.

Als Kriterium für das Ende der Lebensdauer einer Füllung wurde folgendes festgelegt: Es wurde

- eine neue Füllung auf der selben Fläche des selben Zahnes gelegt,

- eine Wurzelbehandlung auf dem entsprechenden Zahn durchgeführt oder

- der Zahn entfernt.

Aufgrund der vorliegenden Daten ist es möglich, die Lebensdauer in Tagen zu berechnen. Für die Schätzung der Überlebensfunktion bzw. der Ausfallrate (mit SAS) wird analog der Abrechnungsperiode die Lebensdauer in Quartalen verwendet.

\section{Deskriptive Analysen}

Die 3.4 Millionen Füllungen verteilen sich auf rund 450000 Patienten, die zwischen 1994 und 2002 mit Füllungen versorgt wurden. Das Geschlechterverhältnis beträgt 50:50. Ein Patient wurde als Stammpatient eingestuft, wenn er maximal einmal im Behandlungsverlauf bei einem anderen Arzt als seinem ursprünglichen Arzt war und eben zu diesem wieder zurückgekehrt ist. 70\% der Personen wurden demnach als Stammpatienten definiert, $30 \%$ als Wechselpatienten klassifiziert.

Seitens der Leistungserbringer ist festzuhalten, dass drei Viertel der Ärzte männlich und ein Viertel weiblich ist. Insgesamt wurden nur Daten von jenen 424 Zahnärzte mit mehr als 150 Füllungsabrechnungen im Beobachtungszeitraum und einer Praxisniederlassung in Oberösterreich in der Analyse berücksichtigt.

Hinsichtlich der Lebensdauer einer Füllung ist anzumerken, dass die Haltbarkeit einer Füllung laut Honorarordnung sich auf mindestens zwei Jahre belaufen muss. Vor Ablauf dieses Zeitraumes kann die Füllung nur mit Angaben von besonderen Gründen erneuert und verrechnet werden (siehe Gesamtvertrag für die Vertragsfachärzte der ZAMUKIHeilkunde, 2005). 


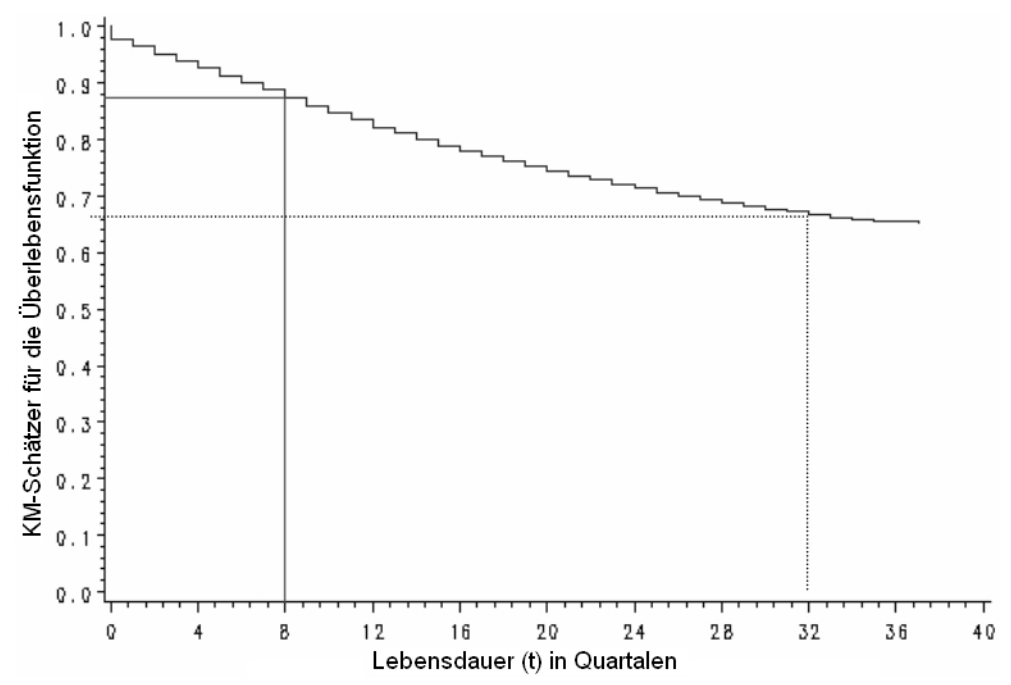

Abbildung 2: Geschätzte Überlebensfunktion aller Seitzahnfüllungen

77.8\% der Lebensdauern sind zensiert, also Füllungen die bis zum 31.12.2002 überlebten, die übrigen 22.2\% sind Ausfälle, d.h. Füllungen die ohne Flächenausdehnung erneuert wurden, an denen eine Wurzelbehandlung durchgeführt oder der Zahn selbst entfernt wurde.

Eine erste Schätzung der Überlebensfunktion $S(t)=P(T>t)$ mittels KaplanMeier-Schätzer (Abbildung 2) ergibt eine Überlebenswahrscheinlichkeit der Füllungen von $87.5 \%$ nach zwei Jahren und $66.7 \%$ nach acht Jahren.

Eine genauere Beschreibung des Ausfallverhaltens als die Überlebensfunktion, bietet die Ausfall- oder Hazardrate

$$
h_{t}\left(t_{0}\right)=\lim _{\Delta_{t} \downarrow 0} \frac{1}{\Delta_{t}} \cdot P\left(t_{0} \leq t<t_{0}+\Delta_{t} \mid t \geq t_{0}\right) .
$$

Abbildung 3 zeigt die nach der Sterbetafelmethode geschätzte Hazardrate der Lebensdauer in Quartalen. Die Hazardrate hat den höchsten Wert im ersten Quartal, d.h. bei Erhalt einer Füllung und weist ein zweites Maximum im neunten Quartal auf.

Auch bei getrennter Analyse der Verweildauer nach den verschiedenen Kovariablen ergibt sich ein ähnliches Bild, so dass von einem systematischen Effekt auszugehen ist. Während das erste Maximum eher auf das Patientenverhalten zurückzuführen ist (Aushärtungszeit wird nicht abgewartet) ist der Grund für das Maximum im neunten Quartal mit der in der Honorarordnung festgelegten Möglichkeit einer Wiederverrechnung nach zwei Jahren zu suchen. Die Wahrscheinlichkeit einer Füllung im ersten Quartal auszufallen liegt bei $2.4 \%$, im neunten Quartal bei $1.7 \%$ und im letzten uns noch zur Verfügung stehenden Quartal bei nur $0.6 \%$.

Die erfreuliche Erkenntnis, dass immerhin zwei Drittel der Füllungen länger als acht Jahre erhalten bleiben und die Ausfallwahrscheinlichkeit auch von alten Füllungen sehr gering ist, wird durch die vertragsbedingte Ausfallspitze im dritten Jahr nach Erhalt einer Füllung getrübt.

Zur Analyse des Einflusses von Kovariablen, wie Geschlecht des Patienten, Region, 


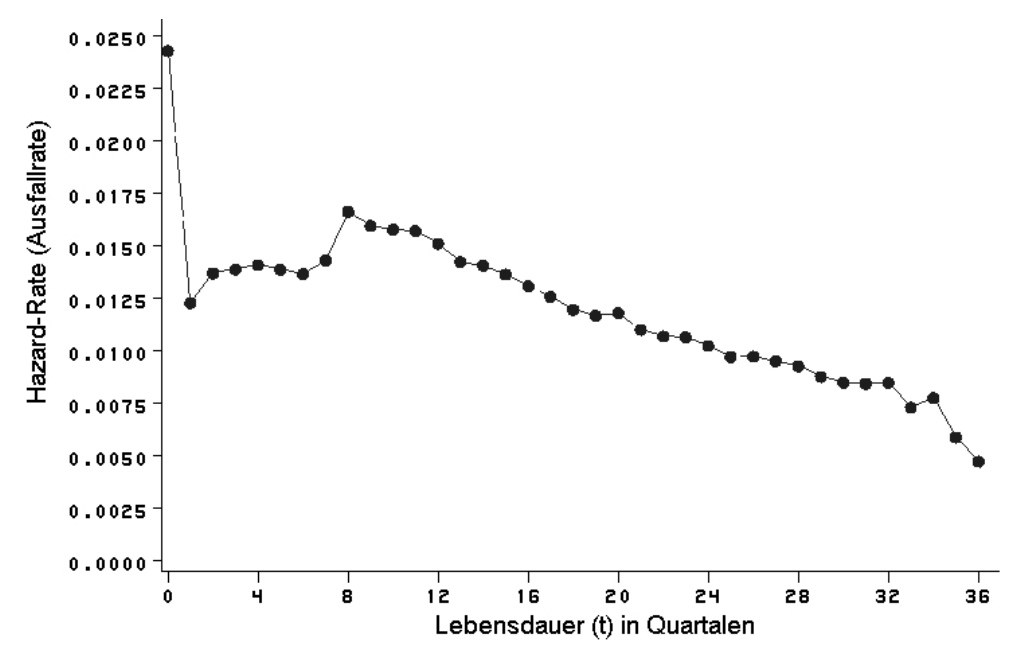

Abbildung 3: Hazardrate in Quartalen

Alter, Stamm-/Wechselpatient oder Geschlecht des Arztes auf die Lebensdauer wurde durch die Hypothese gleicher Hazardraten mittels Log-Rank-Tests überprüft. Auf Grund der großen Datenmenge wurden in unseren Analysen auch sehr kleine und damit nicht relevante Differenzen signifikant.

Deutliche Unterschiede in den Überlebensfunktionen ergeben sich für die Kovariable Praxisgröße nach Anzahl gelegter Füllungen (Abbildung 4). Jene Gruppe von 21 Ärzten (5\%) mit den meisten angefertigten Füllungen pro Jahr (mehr als 1870 Füllungen auf den Seitzähnen) weist die niedrigste Überlebenswahrscheinlichkeit bei Füllungen auf. In dieser Gruppe liegt der Zweijahreswert bei 84.7\% und nach acht Jahren nur bei $58.3 \%$. Hinsichtlich des Geschlechtes des Arztes und Alter des Arztes $(<45$ Jahre, $\geq 45$ Jahre) liegen die beobachteten Unterschiede der Überlebensfunktion bei zwei bis drei Prozentpunkten. Bei einer Einteilung der Daten nach Regionen (Abbildung 5) schneidet das Innviertel signifikant besser ab als alle anderen Regionen, vor allem als der Zentralraum (Linz, Wels, Steyr).

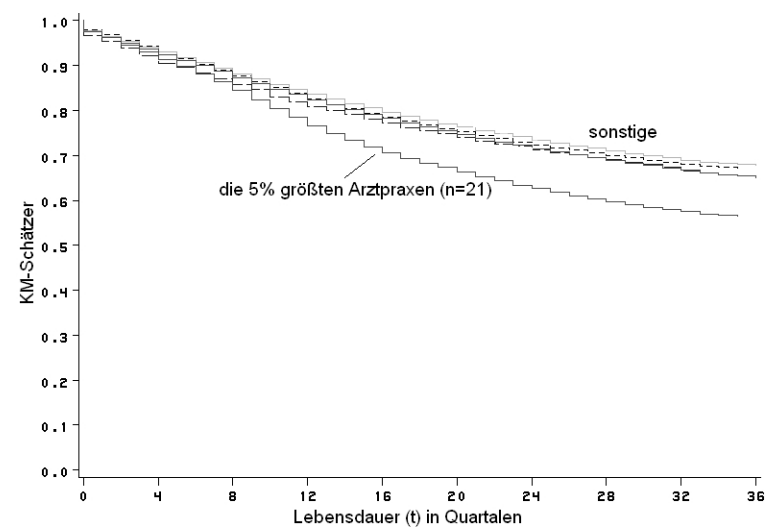

Abbildung 4: Überlebensfunktionen nach Anzahl der Füllungen/Patienten

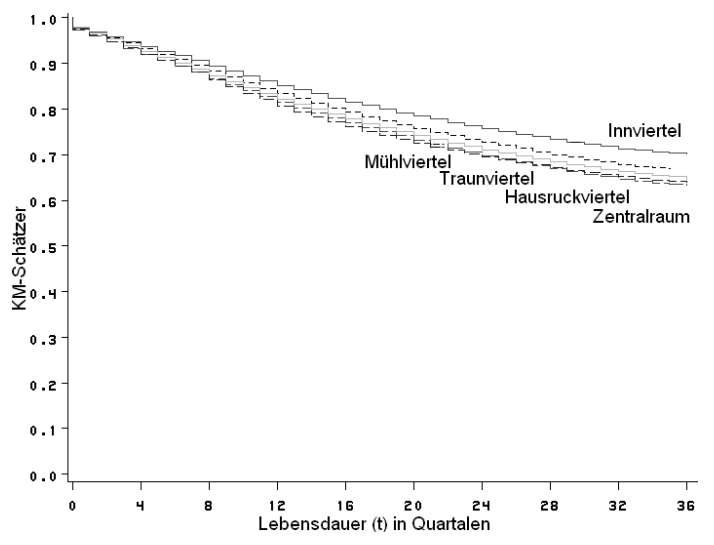

Abbildung 5: Überlebensfunktionen nach Regionen 


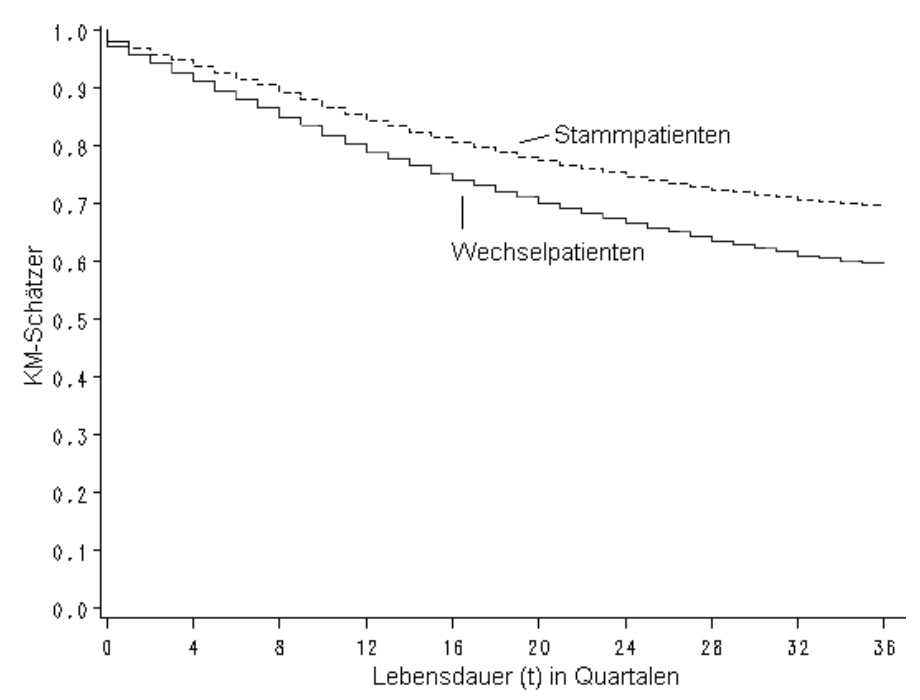

Abbildung 6: Überlebensfunktionen nach Stamm- und Wechselpatienten

Für die Auswertung der Kovariablen Alter der Patienten, Geschlecht der Patienten sowie Patientenstatus ergeben sich nur hinsichtlich des Patientenstatus relevante Unterschiede (Abbildung 6). Die Überlebensfunktion der Füllungen von Stammpatienten verläuft deutlich über jener der Wechselpatienten. Nach acht Jahren liegt die Differenz bei über zehn Prozentpunkten.

Insbesondere für die geringere Lebensdauer bei Wechselpatienten ist die UrsacheWirkungsrelation nicht klar: Einerseits wechselt ein Patient vielleicht deswegen den Zahnarzt, weil er mit der Betreuung unzufrieden ist (z.B. wenn Füllungen schon öfter erneuert werden mussten), und andererseits könnte ein Zahnarzt Füllungen eines zu ihm wechselnden Patienten austauschen, weil er von der Qualität und Haltbarkeit der vorhandenen Füllungen nicht überzeugt ist.

Signifikante Unterschiede in den Haltbarkeiten ergeben sich auch für die Lage des Zahnes (Prämolar/Molar bzw. Zahn 4 und 5/Zahn 6 und 7) (Pfoser et al., 2005) oder der Flächenausdehnung der Füllung. So weisen Einflächenfüllungen im ersten Jahr eine deutlich höhere Ausfallwahrscheinlichkeit auf, ab dem zweiten Jahr liegen die Überlebenswahrscheinlichkeiten von Mehrflächenfüllungen zunehmend unter der von Einflächenfüllungen.

\section{Das Cox-Modell}

Um die simultane Wirkung der Kovariablen auf die Lebensdauer zu untersuchen, verwenden wir das Cox-Modell. Ist $\mathbf{x}=\left(x_{1}, \ldots, x_{k}\right)^{\prime}$ der Kovariablenvektor mit den Ausprägungen $x_{i}$ gleich 0 oder 1 , so wird die Hazardrate als Produkt einer Basishazardrate $h_{0}(t)$ und einer Funktion der Kovariablen $c(\mathbf{x})$ angenommen.

Im Cox-Modell ist die Hazardrate gegeben durch

$$
h_{t}(t \mid \mathbf{x})=h_{0}(t) e^{\mathbf{x}^{\prime} \boldsymbol{\beta}} .
$$


Tabelle 1: Kovariablen und deren Bedeutung im Cox-Modell

\begin{tabular}{lll}
\hline Label & Beschreibung & Ausprägung \\
\hline REGION & Region der Arztpraxis & $1=$ Land, 0=Stadt \\
PRAXGRF & Praxisgröße nach Zahl der Füllungen & $1=$ die größten 5\%, 0=sonst \\
PRAXGRVS & Praxisgröße nach Zahl der Patienten & $1=$ die größten 5\%, 0=sonst \\
ALTGRPVP & Altersgruppe des Arztes & $1 \geq 45$ Jahre, 0 $<45$ Jahre \\
SEXVP & Geschlecht des Arztes & $1=$ weiblich, 0=männlich \\
STATUS & Patientenstatus & $1=$ Stammpat., 0=Wechselpat. \\
LAGE & Lage des Zahnes im Kiefer & $1=$ Prämolar, 0=Molar \\
ZAHN4 & 4-er Zähne & $1=$ Zahn 4, 0=sonst \\
ZAHN6 & 6-er Zähne & $1=$ Zahn 6, 0=sonst \\
POS6 & Honorarposition 6 (Einflächenfüllung) & $1=$ Pos 6, 0=sonst \\
\hline
\end{tabular}

In der Analyse wurden jene Kovariablen berücksichtigt, für deren Überlebensfunktionen sich relevante Unterschiede gezeigt haben. Tabelle 1 gibt eine Übersicht über die im Cox-Modell berücksichtigten Kovariablen.

Die Effekte für die einzelnen Zähne 4 bis 7 ergeben sich aus Kombinationen der Dummyvariablen LAGE, ZAHN4 und ZAHN6. Bezüglich der Füllungspositionen (Flächenausdehnung) zeigen sich nur bei den Einflächenfüllungen (Honorarposition 6) relevante Unterschiede in den Lebensdauern im Vergleich zu den Mehrflächenfüllungen und Höckerdeckungen (Honorarpositionen 7 bis 9). Bei der Modellierung regionaler Effekte haben wir uns für eine Einteilung in Stadt und Land entschieden. Größerer Konkurrenzdruck und mobilere, aufgeklärtere Patienten lassen hier Unterschiede erwarten. Die Tabelle 2 zeigt die wichtigsten Ergebnisse eines Cox-Modells mit schrittweiser Aufnahme der Kovariablen.

Wie nicht anders zu erwarten, wurden ob der großen Datenmenge alle Variablen in das Modell aufgenommen. Entgegen unseren Vermutungen ist der Einfluss des regionalen Effektes gering, wobei die Ausfallrate für Praxen auf dem Land marginal niedriger ist als für jene in der Stadt.

Ein sehr starker Effekt ergibt sich für die Praxisgröße. In Arztpraxen mit vielen Füllungen (PRAXGRF) liegt die Ausfallrate um knapp ein Drittel über derer anderer Praxen. Hier könnte Zeitmangel bei der Füllungslegung der Grund für die geringeren Haltbarkeiten sein. Auch die Variable Praxisgröße nach Anzahl der Patienten (PRAXGRVS) weist in diese Richtung, so dass eine Senkung der Patientenzahl und damit einhergehend eine Senkung des Zeitdrucks die Qualität und Lebensdauer der Füllungen erhöhen könnte.

Hinsichtlich des Patientenstatus (STATUS) zeigt sich, dass die Ausfallrate der Stammpatienten nur rund $70 \%$ jener der Wechselpatienten beträgt.

Bezüglich der Lage des Zahnes (LAGE) ergibt sich, dass Prämolare (Zähne 4 und 5) ein um 20\% geringeres Ausfallrisiko aufweisen als Molare (Zähne 6 und 7). Grund hierfür kann die stärkere Kaubelastung auf den hinteren Backenzähnen (Molare) sein. Innerhalb der Prämolaren unterscheiden sich Zahn 4 und Zahn 5 kaum, während innerhalb der Molaren ein deutlicher Unterschied zwischen Zahn 6 und Zahn 7 erkennbar ist. Die um $27 \%$ höhere Ausfallrate der 6er Zähne lässt sich physiologisch erklären. Bei den zweiten Zähnen brechen die 6er Zähne als erste im Kiefer durch und müssen somit früher 
Tabelle 2: Parameterschätzer und Hazard-Ratio der Regressionsmodelle, Standardfehler in Klammern, Fehlerniveau $\alpha=0.05$, stepwise im Cox-Modell

\begin{tabular}{|c|c|c|c|c|c|c|}
\hline \multirow[b]{2}{*}{ Label } & \multicolumn{2}{|c|}{$\begin{array}{c}\text { Cox-Modell } \\
N=3364344\end{array}$} & \multicolumn{2}{|c|}{$\begin{array}{l}\text { Cox-Modell } \\
N=42400\end{array}$} & \multicolumn{2}{|c|}{$\begin{array}{l}\text { Frailty-Modell } \\
\quad N=42400\end{array}$} \\
\hline & $\begin{array}{c}\hat{\beta} \\
(S E)\end{array}$ & Ratio & $\begin{array}{c}\hat{\beta} \\
(S E)\end{array}$ & Ratio & $\begin{array}{c}\hat{\beta} \\
(S E)\end{array}$ & Ratio \\
\hline REGION & $\begin{array}{l}-0.061 \\
(0.002)\end{array}$ & 0.941 & $n s$ & - & - & - \\
\hline PRAXGRF & $\begin{array}{c}0.270 \\
(0.005)\end{array}$ & $\begin{array}{l}1.310 \\
1.310\end{array}$ & $\begin{array}{c}0.287 \\
(0.052)\end{array}$ & 1.333 & $\begin{array}{c}0.267 \\
(0.101)\end{array}$ & 1.306 \\
\hline PRAXGRVS & $\begin{array}{c}0.097 \\
(0.005)\end{array}$ & 1.102 & $\begin{array}{c}0.159 \\
(0.056)\end{array}$ & 1.172 & $\begin{array}{l}0.170^{n s} \\
(0.106)\end{array}$ & 1.186 \\
\hline ALTGRPVP & $\begin{array}{c}0.147 \\
(0.002)\end{array}$ & 1.158 & $\begin{array}{c}0.156 \\
(0.021)\end{array}$ & 1.169 & $\begin{array}{c}0.137 \\
(0.033)\end{array}$ & 1.147 \\
\hline SEXVP & $\begin{array}{c}0.086 \\
(0.003)\end{array}$ & 1.089 & $\begin{array}{c}0.106 \\
(0.023)\end{array}$ & 1.112 & $\begin{array}{c}0.094 \\
(0.045)\end{array}$ & 1.099 \\
\hline STATUS & $\begin{array}{l}-0.347 \\
(0.002)\end{array}$ & 0.707 & $\begin{array}{l}-0.424 \\
(0.021)\end{array}$ & 0.654 & $\begin{array}{l}-0.436 \\
(0.022)\end{array}$ & 0.646 \\
\hline LAGE & $\begin{array}{l}-0.227 \\
(0.003)\end{array}$ & 0.797 & $\begin{array}{l}-0.284 \\
(0.027)\end{array}$ & 0.753 & $\begin{array}{l}-0.287 \\
(0.027)\end{array}$ & 0.750 \\
\hline ZAHN4 & $\begin{array}{l}-0.062 \\
(0.004)\end{array}$ & 0.940 & $n s$ & - & - & - \\
\hline ZAHN6 & $\begin{array}{c}0.241 \\
(0.003)\end{array}$ & 1.272 & $\begin{array}{c}0.173 \\
(0.026)\end{array}$ & 1.189 & $\begin{array}{c}0.166 \\
(0.026)\end{array}$ & 1.181 \\
\hline POS6 & $\begin{array}{l}-0.172 \\
(0.003) \\
\end{array}$ & 0.842 & $\begin{array}{l}-0.109 \\
(0.024) \\
\end{array}$ & 0.897 & $\begin{array}{l}-0.117 \\
(0.025) \\
\end{array}$ & 0.890 \\
\hline Frailty $(\sim \Gamma(\theta, 1))$ & - & - & - & - & $\begin{array}{c}\hat{\theta}=0.110 \\
(0.011)\end{array}$ & - \\
\hline$\overline{R^{2}}$ & 2.19 & & 2.13 & & $5.10^{c}$ & \\
\hline
\end{tabular}

ns ... nicht signifikant

mit Füllungen versorgt werden. Die Zahnsubstanz nimmt nach jeder Erneuerung einer Füllung ab, was in Folge zu einer schlechteren Haltbarkeit führen kann.

\section{Das Shared-Frailty-Modell}

Wie in vielen Lebensdaueranalysen muss auch für die vorliegenden Daten davon ausgegangen werden, dass unbeobachtete Heterogenität vorliegt, d.h. dass nicht alle Einflussfaktoren für die Lebensdauer von Zahnfüllungen beobachtet wurden und diese in der Population unterschiedlich sind.

Ist eine Population hinsichtlich unbeobachteter Einflussfaktoren homogen, so entspricht die Hazardrate jedes Individuums jener der Gesamtpopulation. Die Hazardrate für eine heterogene Population ergibt sich jedoch durch Mischung der individuellen Verteilungen und lässt keinen Schluss auf den Verlauf einer individuellen Hazardrate zu. 
Vaupel, Manton, and Stellard (1979) schlug daher vor, einen multiplikativen Term $\omega$, die sogenannte Frailty, der diese unbeobachteten Faktoren zusammenfasst, in die Hazardrate einzuführen, d.h. folgendes Modell für die Ausfallrate zu verwenden

$$
h_{t}(t \mid \mathbf{x}, \omega)=\omega \cdot h_{0}(t) e^{\left(\mathbf{x}^{\prime} \boldsymbol{\beta}\right)}=h_{0}(t) e^{\left(\mathbf{x}^{\prime} \boldsymbol{\beta}+\log (\omega)\right)} .
$$

$\exp \left(\mathbf{x}^{\prime} \boldsymbol{\beta}\right)$ kann als Effekt der beobachteten Faktoren und $\omega$ als Effekt der unbeobachteten Heterogenität auf die Hazardrate interpretiert werden.

Für die Lebensdauer der Zahnfüllungen ist von Interesse, ob es entsprechend einem Zentrumseffekt in klinischen Studien (Glidden und Vittinghoff, 2004) einen Arzteffekt gibt. Solche Arzteffekte führen zu einer Clusterung der Lebensdauern, d.h. Abhängigkeiten zwischen den Lebensdauern der Füllungen eines Arztes. Nichtberücksichtigen der Clusterung kann zu irreführenden Schlüssen bezüglich der Kovariableneffekte führen.

Für die gegenständliche Analyse ist ein Arzteffekt auch für sich von Interesse, erlaubt eine Schätzung des Frailtywertes für den einzelnen Arzt doch eine statistische Beurteilung der Qualität der erbrachten Leistung. Analog zu Glidden and Vittinghoff (2004) wurde als Clustervariable die Arztnummer festgelegt und zur Schätzung der individuellen Effekte der Ärzte ein Gamma-Shared-Frailty-Modell verwendet, d.h. die Füllungen eines Arztes teilen sich eine Frailty $\left(\omega_{i}, i=1, \ldots, 424\right)$.

Um festzustellen, ob unbeobachtete, dem Arzt zuzuordnende, Heterogenität in den Daten zu finden ist, wurde im Rahmen einer Pilotstudie für jeden der 424 Ärzte eine Zufallsstichprobe von $n=100$ Füllungen gezogen. Mit diesen 42400 Datensätzen wurde in SAS und R zunächst das Cox-Modell abermals geschätzt, um die Güte der Stichprobe zu überprüfen und anschließend das Gamma-Shared-Frailty-Modell (in R) anzupassen.

\section{Die Güte der Modelle}

Die Schätzungen der Parameter des Cox-Modells zeigen für die Stichprobe eine gute Übereinstimmung mit jenen des vollständigen Datensatzes.

Die Effekte der Kovariablen REGION und ZAHN4, die im vollständigen Datensatz mit Hazard-Ratios von jeweils 0.94 - am schwächsten waren, sind für den Stichprobendatensatz nicht signifikant. Diese beiden Kovariablen wurden in den weiteren Analysen nicht mehr in die Modelle aufgenommen.

Die Schätzer der Effekte für die übrigen Kovariablen sind in Spalte 3 und 4 von Tabelle 2 angegeben. Sie stimmen sowohl in Richtung als auch in Größe weiterhin gut mit denen des auf allen Daten basierenden Modells (Tabelle 2, Spalte 1 und 2) überein.

Bei guter Modellanpassung sollte die kumulierte Hazardrate der Cox-Snell-Residuen im Plot gegen die Cox-Snell-Residuen einer $45^{\circ}$-Geraden folgen. Wie Abbildung 7 zeigt, trifft dies für das Cox-Modell des Stichprobendatensatzes zu.

Allerdings erklärt das Cox-Modell nur $2.13 \%$ der Variation $\left(R^{2}=1-\left(L_{r} / L_{u}\right)^{2 / n}\right)$. Hierbei bezeichnen $L_{r}$ und $L_{u}$ die Likelihoods des Modells ohne (restricted) und mit (unrestricted) Kovariablen. Nach Schemper and Stare (1996) sind kleine Bestimmtheitsmaße in Lebensdaueranalysen, insbesondere in Cox-Modellen nicht ungewöhnlich, zudem sind im vorliegenden Fall bezogen auf die Größe des Datenbestandes nur wenige Kovariablen vorhanden. 


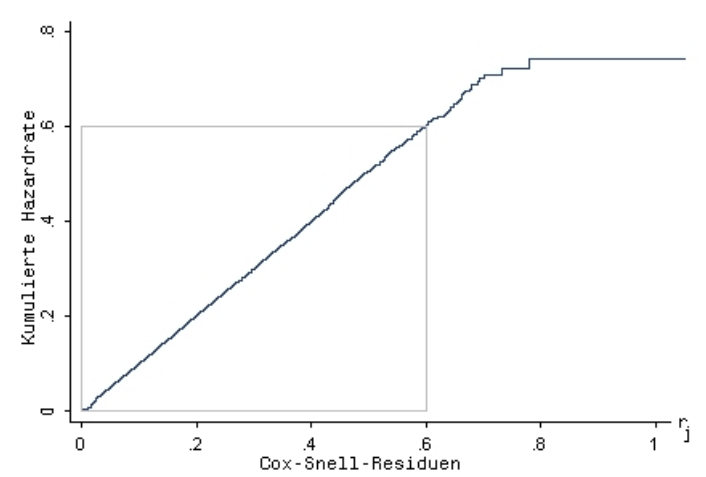

Abbildung 7: Cox-Snell-Residuen des Cox-Modells

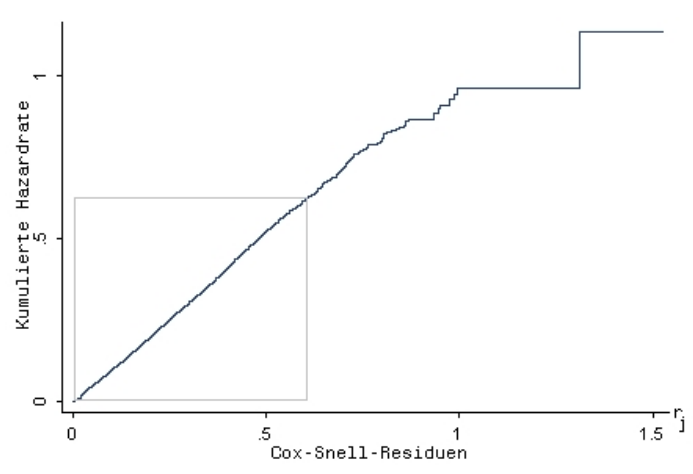

Abbildung 8: Cox-Snell-Residuen des Frailty-Modells

Tabelle 3: Kennzahlen der Frailty-Werte

\begin{tabular}{lr|ll}
\hline$n$ & 424 & Min & 0.416 \\
Mittelwert & 1.0 & Max & 2.185 \\
Median & 0.977 & Range & 1.770 \\
25\%-Perzentil & 0.798 & Std.-Abw. & 0.281 \\
75\%-Perzentil & 1.156 & Varianz & 0.079 \\
\hline
\end{tabular}

Das Shared-Frailty-Modell zur Bestimmung der Arzteffekte wurde mit den selben Kovariablen wie das Cox-Modell für die Daten der Stichprobe geschätzt. Die Parameterschätzer sind in Tabelle 2, Spalte 5 bis 6 angegeben. Die Stärke der Effekte wird ähnlich wie im Cox-Modell geschätzt, der Effekt der Praxisgröße nach Zahl der Patienten (PRAXGRVS) ist jedoch nicht mehr signifikant auf dem 5\%-Niveau. Die Cox-Snell-Residuen in Abbildung 8 zeigen ebenfalls eine gute Modellanpassung an. Durch die Aufnahme der Frailty-Variablen erhöht sich das Bestimmtheitsmaß auf 5.1\%.

\section{Arzteffekte in der Füllungstherapie}

Abbildung 9 stellt die Verteilung der Frailty-Werte aller 424 Ärzte dar. Frailty-Werte um 1 definieren "Standardärzte". Größere Frailty-Werte weisen erhöhte Schwäche, also geringere Haltbarkeiten auf und Werte kleiner 1 geringere Schwäche, demzufolge also niedrigere Ausfallraten.

Ausgehend vom Standardarzt mit Frailty-Wert $\omega_{i}=1$ und Kovariablenausprägungen $x_{i}=0$ betrachten wir die Überlebenswahrscheinlichkeiten nach zwei und neun Jahren. Nach zwei Jahren liegt diese für den Standardarzt bei 86\%, für den besten Arzt - d.h. jenem mit dem geringsten Frailty-Wert - bei 94\% (Abbildung 10). Für den Arzt mit dem größten Frailty-Wert erhält man hingegen nur einen Wert von $72 \%$. Nach neun Jahren ergibt sich eine Überlebenswahrscheinlichkeit von $61 \%$ für den Standardarzt bzw. $81 \%$ für den Arzt mit der geringsten und $34 \%$ für den Arzt mit der höchsten Frailty. 


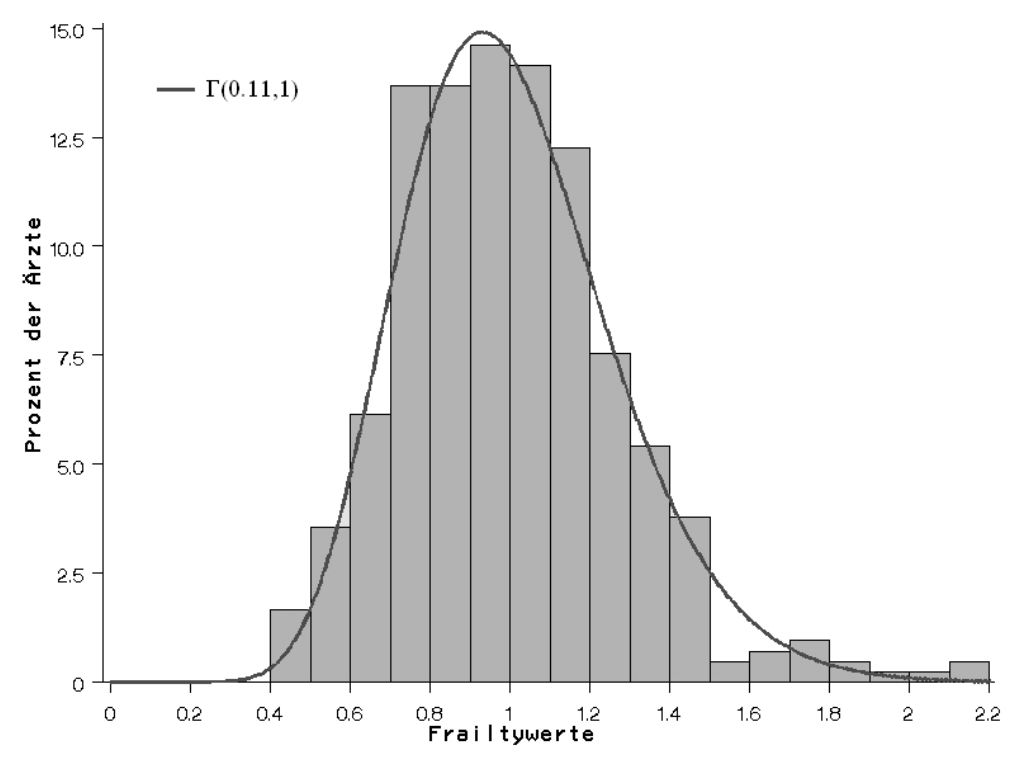

Abbildung 9: Histogramm der Frailty-Werte, $\hat{\omega}_{i} \sim \Gamma(0.11,1)$

Das Shared-Frailty-Modell zeigt den arztindividuellen Beitrag zur Haltbarkeit seiner Füllungen, wobei Effekte durch die in Tabelle 2 angeführten Kovariablen als Begründung unterschiedlicher Haltbarkeiten ausscheiden. Damit gelingt eine Segmentierung der Leistungserbringer hinsichtlich ihrer Lebensdauern der gelegten (eigentlich: abgerechneten) Füllungen. Schaltet man alle vorhandenen Kovariableneffekte aus so zeigt sich, dass der Arzt selbst noch in einem großen Ausmaß für die Haltbarkeit der von ihm erstellten Füllungen verantwortlich ist. Diese Erkenntnis mag nicht überraschen, mit Hilfe des Frailty-Modells kann dieses Ausmaß jedoch quantifiziert und zu Qualitätsvergleichen herangezogen werden.

Die Streuung der Frailty-Werte bei gleichen (oder ähnlichen) Überlebenswahrscheinlichkeiten lässt eine vom Niveau der Überlebenswahrscheinlichkeit abhängigen Bewertung des Arzteffektes zu. Um dies zu veranschaulichen wurden in Abbildung 11 für die Daten der Stichprobe die mittels Kaplan-Meier-Schätzer berechneten 5 Jahres-Überlebenswahrscheinlichkeiten in einem Streudiagramm gegen die Frailties aufgetragen. Die Spannweite der Frailty-Werte ist mit 1.77 überraschend hoch (siehe Tabelle 3). Die Abbildung 11 zeigt, dass bis auf wenige Ausnahmen bei jedem Niveau der Überlebenswahrscheinlichkeit einer Füllung nach 5 Jahren in den Frailties Spannweiten von rund 0.8 auftreten. So unterscheiden sich beispielsweise für Ärzte mit einer 5-Jahres-Überlebenswahrscheinlichkeit von $80 \%$ die Frailty-Werte um bis zu $140 \%$.

Das bedeutet aber, dass die Überlebenswahrscheinlichkeit der Füllungen, die ein bestimmter Arzt erzielt, nur zu einem Teil auf die Effekte von Kovariablen zurückzuführen sind und sich zu einem erheblichen Teil durch individuelle Effekte ergeben. Eine Honorierung nur aufgrund erzielter Überlebenswahrscheinlichkeiten wäre insofern nicht gerechtfertigt, als auch Ärzte mit hoher Frailty gute Überlebenswahrscheinlichkeiten erzielen können, wenn die Kovariablenstruktur günstig ist. 


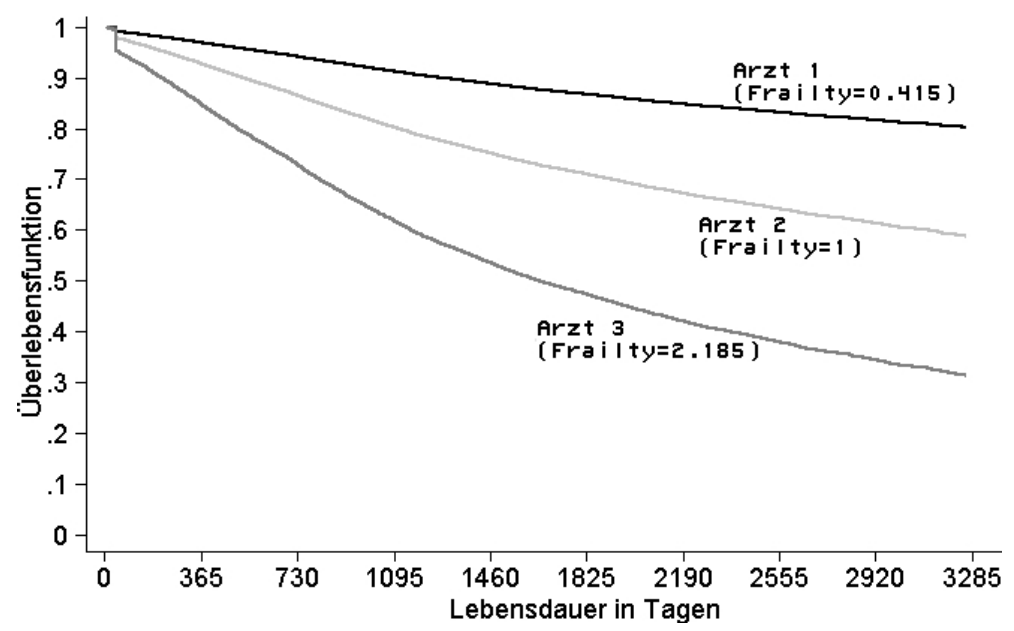

Abbildung 10: Basis-Überlebensfunktion $S_{0}(t)($ Standardarzt mit Frailty-Wert $=1)$ und Überlebensfunktionen der Ärzte mit dem kleinsten $S_{0}(t)^{0.415}$ und dem größten FrailtyWert $S_{0}(t)^{2.185}$

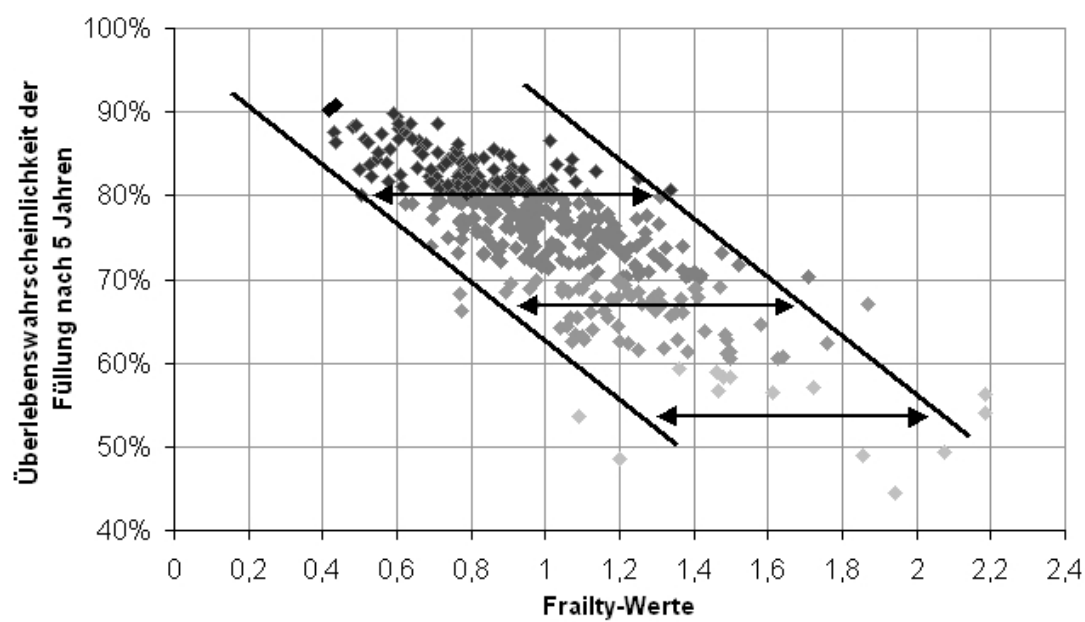

Abbildung 11: Streudiagramm der KM-Schätzer nach 5 Jahren und Frailty-Werte für alle $n=424$ Ärzte

\section{Schlussfolgerungen}

Unsere Analyse basiert auf breitem Datenmaterial. Sowohl das betrachtete Ausmaß von neun Jahren als auch die Anzahl der gelegten Füllungen lassen einen sicheren Schluss auf die unter Praxisbedingungen laufende Füllungstherapie zu. Daraus abgeleitet können wesentliche Ansatzpunkte für eine Verbesserung der vertraglichen Ausgestaltung der Füllungstherapie gefunden werden.

1. Jene Ärzte mit den meisten gelegten Füllungen weisen die geringsten Haltbarkeiten auf. Hier wäre eine Senkung der Frequenz (oder der Patientenzahl) sinnvoll, um den Zeitdruck bei der Füllungslegung zu vermindern und die Qualität und die Lebensdauer der Füllungen zu erhöhen. 
2. Die geforderte Mindesthaltbarkeit von zwei Jahren führt zu Ausfallspitzen im dritten Jahr, wiewohl wir wissen, dass die Überlebenswahrscheinlichkeit nach 2 Jahren bei $87.5 \%$ liegt und nach 8 Jahren immerhin noch bei $66.7 \%$. Internationale Studien (vgl. obige Quelle, v.a. Smales und Hawthorne, 1997) haben gezeigt, dass Füllungen deutlich länger halten können, weshalb eine Anhebung der Mindesthaltbarkeit vertretbar erscheint.

3. Nachdem wir festgestellt haben, dass es zwischen Arzt-Patientenbindung und Haltbarkeit von Füllungen einen Zusammenhang gibt, könnten Bemühungen des Zahnarztes, seine Patienten durch Aufklärung, Service, Kundenorientierung u.ä. zu binden, positiv honoriert werden.

4. Wie die Verteilung der Frailty-Werte zeigt, hat der Arzt selbst Einfluss auf die Haltbarkeit seiner gelegten Füllungen. Im Sinne einer anreizorientierten Honorierung qualitativ guter Füllungstherapie könnten nun Ärzte mit niedrigeren Frailty-Werten höhere Honorare erhalten.

\section{Literatur}

Gesamtvertrag für die Vertragsfachärzte der ZAMUKI-Heilkunde. (2005). Abgeschlossen zwischen Ärztekammer und Hauptverband der österreichischen Sozialversicherungsträger.

Glidden, D. V., und Vittinghoff, E. (2004). Modelling clustered survival data from multicenter clinical trials. Statistics in Medicine, 23, 369-388.

Pfoser, N., Malek, L., Schableger, K., und Bukal, J. (2005). Statistische Bewertung der Verweildauer von Zahnfüllungen in Oberösterreich. Stomatologie, 102, 177-182.

Schemper, M., und Stare, J. (1996). Explained variation in survival analysis. Statistics in Medicine, 15, 1999-2012.

Smales, R. J., und Hawthorne, W. S. (1997). Long-term survival of extensive amalgams and posterior crowns. Journal of Dentistry, 25, 225-227.

Statistisches Handbuch der österreichischen Sozialversicherung. (2005). Hrsg. Hauptverband der österreichischen Sozialversicherungsträger, Wien.

Vaupel, J. W., Manton, K. G., und Stellard, E. (1979). The impact of heterogeneity in individual frailty on the dynamics of mortality. Demography, 16, 439-454.

Anschrift der Autoren:

$\begin{array}{ll}\text { Karl Schableger } & \text { Helga Wagner } \\ \text { Notburga Pfoser } & \text { Institut für Angewandte Statistik } \\ \text { OÖ Gebietskrankenkasse } & \text { Johannes-Kepler-Universität Linz } \\ \text { Gruberstraße } 77 & \text { Altenbergerstraße 69 } \\ \text { 4020 Linz } & 4040 \text { Linz } \\ \text { Österreich } & \text { Österreich } \\ \text { E-mail: karl.schableger@ooegkk.at } & \text { E-mail: helga.wagner@jku .at } \\ \quad \text { notburga.pfoser@ooegkk.at } & \end{array}$

